\title{
Retinopathy from Interferon Therapy for Cutaneous Melanoma in a Patient with Hypertension and Diabetes-A Case Report
}

\author{
Alice $\mathrm{Y}$ Kim, BS, ${ }^{1}$ Meena George, MD, $\mathrm{PhD}^{2}$ and Jesse L Berry, $\mathrm{MD}^{3}$
}

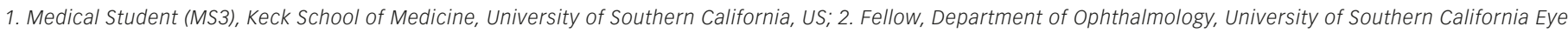

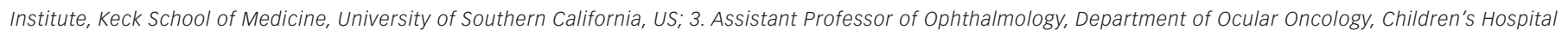

Los Angeles, University of Southern California Eye Institute, Keck School of Medicine, University of Southern California, US

\begin{abstract}
High-dose pegylated interferon alpha-2b (IFN $\alpha 2 \mathrm{~b}$ ) is an antineoplastic agent and biological response modulator used as first-line adjuvant therapy in cases of melanoma. Development of IFN-associated retinopathy is a rare but known complication of treatment. In the setting of additional co-morbidities, such as diabetes or hypertension, the etiology of retinopathy may be difficult to assess given the shared findings of nerve fiber layer infarcts and variably affected visual acuity. Thus, it is recommended to conduct a baseline ophthalmologic examination for patients with other comorbidities prior to initiating high-dose IFN therapy to help guide appropriate intervention should retinopathy develop. Once detected, the distribution of nerve fiber layer infarcts should be noted on exam, as well as duration and severity of diabetes, hypertension, and IFN therapy to help narrow the differential. We report a case of a 55-year-old female who developed retinopathy after initiating high-dose IFN therapy for cutaneous melanoma in the setting of a history of diabetes and systemic hypertension. The retinopathy resolved with discontinuation of the adjuvant therapy.
\end{abstract}

\section{Keywords}

Pegylated interferon, retinopathy, hypertension, diabetes, melanoma, nerve fiber layer infarcts, cotton wool spots

Disclosure: Alice Y Kim, BS, Meena George, MD, PhD, and Jesse L Berry, MD, have no relevant conflicts of interest to declare. No funding was received in the publication of this article.

Open Access: This article is published under the Creative Commons Attribution Noncommercial License, which permits any noncommercial use, distribution, adaptation, and reproduction provided the original author(s) and source are given appropriate credit.

Compliance with Ethical Guidance: Procedures were followed in accordance with the responsible committee on human experimentation and with the Helsinki Declaration of 1975 and subsequent revisions. Informed consent was received from the patient involved in the report.

Received: July 4, 2015 Accepted: September 24, 2015 Citation: US Ophthalmic Review, 2015;8(2):132-4 DOI: http://doi.org/10.17925/USOR.2015.08.02.132

Correspondence: Meena S George, MD, PhD, Department of Ophthalmology, USC Eye Institute, 1450 San Pablo St, Los Angeles, CA 90089, US. E: Meena.George@med.usc.edu

A 55-year-old woman presented to us for her initial visit with blurry vision for 1 month. She denied any prior ocular history, including diabetic retinopathy. Medical history was significant for diabetes mellitus, hypertension, renal cell carcinoma, and recently resected metastatic malignant melanoma (pT4bN1b, Stage IIIC). Following resection, she was started on high-dose pegylated interferon alpha-2b (IFN $\alpha 2 b$ ) as adjuvant therapy. At this time, she had a glycated hemoglobin $\left(\mathrm{HbA}_{1 \mathrm{c}}\right)$ of $7.4 \%$ with blood pressures ranging from 139 to $153 / 60$ to 87 , for which she took metformin, glipizide, simvastatin, and benazepril. After a month of treatment with IFN, she began noticing blurry vision in both eyes as well as increased floaters, which persisted for 1 month prior to presentation.

On examination, best corrected visual acuity was 20/30-1 in her right eye and 20/40-2 in her left eye. Slit lamp examination was unremarkable. Dilated fundus examination revealed large peripapillary and macular nerve fiber layer infarcts in both eyes. The vessels were within normal limits without tortuosity or sheathing; there were no choroidal masses, hemorrhages, or subretinal fluid. Optical coherence tomography (OCT) showed areas of infarct in the macula bilaterally without macular edema (see Figure 1).

Given the onset of retinopathy following initiation of IFN therapy as well as the size and peripapillary distribution of the nerve fiber layer infarcts, the diagnosis made was IFN-associated retinopathy. IFN treatment was discontinued by her oncology team due to other comorbidities in addition to the retinopathy. On follow-up evaluation 3 months later, her visual acuity improved to 20/20-1 and 20/25. On examination, the regions of infarct had resolved, and OCT confirmed improvement in the nerve fiber layer. After 6 months of IFN cessation, fundus examination showed complete resolution of infarction and rare intraretinal hemorrhages (see Figure 2). Informed consent was received from the patient involved in the study.

\section{Discussion}

High-dose IFN $\alpha 2 b$ is an antineoplastic agent and biological response modulator used as first-line adjuvant therapy in cases of melanoma. ${ }^{1}$ 
Retinopathy is a rare but known complication of IFN therapy. ${ }^{2}$ Though the exact pathogenesis remains unclear, several theories have been proposed including IFN-induced immunologic and endothelial dysregulation leading to occlusions in the retinal microcirculation and formation of nerve fiber layer infarcts and/or retinal hemorrhages. ${ }^{2}$

Studies investigating the risk factors for development of IFN retinopathy have identified diabetes and hypertension as potentially important contributors to retinal pathology given their deleterious effect on the retinal microcirculation. ${ }^{3-5}$ This presents a common conundrum for ophthalmologists who have patients that present for the first time with multiple comorbidities that may each cause retinopathy, as in this patient. Thus, detailed clinical history and examination can be critical in determining the primary cause of retinopathy. Onset of retinopathy within 2 weeks to 3 months following initiation of IFN therapy increases the likelihood of IFN-associated retinopathy. ${ }^{6,7}$ However, this determination is difficult to make by history alone in patients with concurrent diabetes and/or hypertension and no prior ocular exam.

In such cases, dilated fundus examination may further support one diagnosis over the other based on the presence and distribution of various retinal changes. IFN-associated retinopathy has been found to present more commonly with retinal nerve layer infarcts in the peripapillary and posterior pole distribution., ${ }^{3,8-10}$ Other ocular findings include ischemic microvascular changes, microaneurysms, and cystoid macular edema, which are less specific. ${ }^{2,6}$ Infarcts, also known as cotton wool spots, may also develop in diabetic retinopathy, but the presence of microaneurysms, dot and blot hemorrhages, venous beading, intraretinal microvascular abnormalities, and neovascularization may further increase suspicion for diabetic retinopathy over other etiologies. ${ }^{10-12}$ Similarly, hypertensive retinopathy may present with infarct but show additional retinal changes, such as arteriolar narrowing, arteriovenous nicking, hemorrhages, and papilledema..13-15 on exam, our patient demonstrated large peripapillary and macular nerve fiber layer infarcts in both eyes without evidence of retinal vessel compromise or edema, further lending support to our diagnosis of IFN-associated retinopathy over diabetic or hypertensive retinopathy.

Currently, there are no guidelines regarding the re-initiation of IFN therapy in patients who have experienced retinopathy, as there is an unknown and variable risk for recurrent retinopathy. ${ }^{2,7}$ IFN therapy offers a potentially life-saving therapy for patients, and studies have found that most patients with IFN-associated retinopathy are either asymptomatic or have mild visual impairment that is reversible with cessation of IFN. ${ }^{2,7}$ Cases of severe vision loss are rare. ${ }^{2}$ Therefore, the decision to stop treatment should be done in discussion with oncology and considering the complete clinical situation.

Physicians should advise patients of possible visual complications from high-dose IFN, and prompt referral to a retinal specialist is warranted with the onset of any visual symptoms after initiating treatment. Patients with conditions associated with microvascular complications such as diabetes and hypertension may be at further risk for developing retinopathy with IFN administration due to pre-existing damage to the retinal vasculature..$^{3-5}$ For patients with these co-morbidities, we recommend that the oncology team refer the patient for at least one baseline assessment of retinal
Figure 1: At Presentation-Color Fundus Photography with Retinopathy and Optical Coherence Tomography of the Fovea
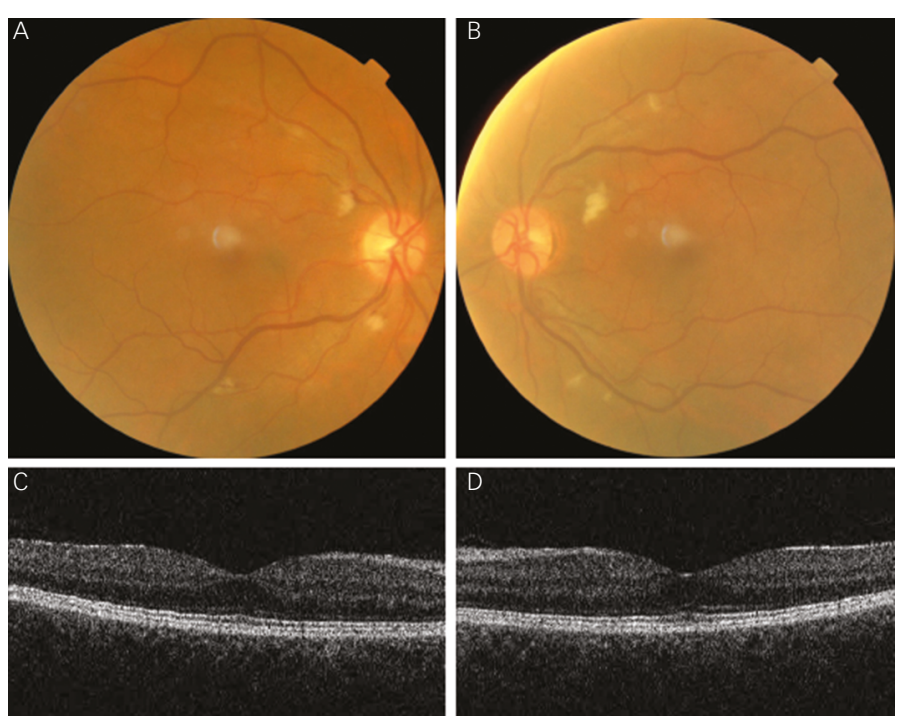

Color fundus photographs showing large peripapillary and posterior pole nerve fiber layer infarcts, right eye $(A)$ and left eye (B) following 2 months of interferon therapy. Optical coherence tomography at the fovea, right eye (C), and left eye (D) with no evidence of macular edema.

\section{Figure 2: Follow-up-Resolution of Retinopathy on Color Fundus Photography and Optical Coherence Tomography with Discontinuation of Interferon}
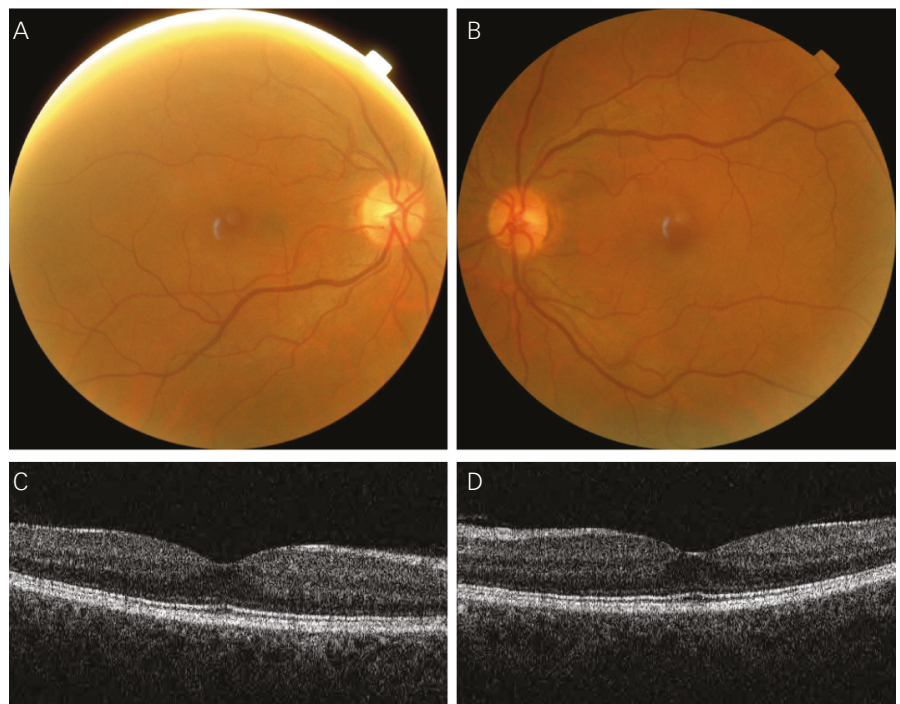

Color fundus photographs showing complete resolution of nerve fiber layer infarcts in the fundus of both eyes ( $A$ and $B$ ) with rare intraretinal hemorrhages in the left eye (B), 6 months after cessation of interferon. Optical coherence tomography at fovea, right eye (C), and left eye (D) with no evidence of intraretinal or subretinal fluid.

health prior to receiving IFN therapy. Further monitoring and frequency of follow-up exams can then be determined by the ophthalmologist and communicated to the oncology team based on the findings on dilated fundus examination and OCT imaging. If retinopathy develops, the timeline and distribution of retinal lesions should be documented and monitored in relation to the patient's glycemic and blood pressure control, with discussion of possible discontinuation of IFN therapy if vision loss worsens or involves the macula. 
1. Kirkwood JM, Strawderman MH, Ernstoff MS, et al., Interferon alfa-2b adjuvant therapy of high-risk resected cutaneous melanoma: The Eastern Cooperative Oncology Group Trial EST 1684, I Clin Oncol, 1996;14:7-17.

2. Monzon J, Hammad N, Stevens S, Dancey J, Retinopathy associated with adjuvant high-dose interferon- $2 b$ in a patient with resected melanoma: a case report and review of the literature, The Oncologist, 2012;17:384-7.

3. Hayasaka S, Nagaki Y, Matsumoto M, Sato S, Interferon associated retinopathy, $\mathrm{Br} /$ Ophthalmol 1998:82:323-5.

4. Panetta JD, Gilani N, Interferon-induced retinopathy and its risk in patients with diabetes and hypertension undergoing treatment for chronic hepatitis $\mathrm{C}$ virus infection, Aliment Pharmacol Ther, 2009;30:597-602.
5. Xue JH, Zhu HH, Wang J, Chen Z, Interferon-associated retinopathy risk in patients with diabetes and hypertensive hepatitis C, World J Gastroenterol, 2014;20:7505-13.

6. Guyer D, Interferon-associated retinopathy, Arch Ophthalmol, 1993;111:350.

7. Fragoso $Y$, Paggiaro M, Mastromauro R, et al., Literature systematic review on the ophthalmological side effects of interferons, Arq Bras Oftalmol, 2011;74:306-10.

8. Wes A, Hong ES, Oetting TA, Interferon-associated retinopathy: communicating with internal medicine, 2010. Available at: http://www.EyeRounds.org/cases/116-Interferon-Retinopathy. htm (accessed July 26, 2010).

9. Esmaeli B, Koller C, Papadopoulos N, Romaguera J, Interferoninduced retinopathy in asymptomatic cancer patients, Ophthalmology, 2001;108:858-60.
10. Schulman J, Liang C, Kooragayala L, King J, Posterior segment complications in patients with Hepatitis C treated with
interferon and ribavirin, Ophthalmology, 2003:110:437-41.

11. Viswanath $K$, McGavin D, Diabetic retinopathy: clinical findings and management, Community Eye Health, 2003;16:21-4.

12. Frank RN, Diabetic retinopathy, N Eng/ J Med, 2004;350:48-58

13. Wagener H, Clay G, Gipner J, Classification of retinal lesions in the presence of vascular hyptertension, Ophthalmol Trans Am SCi, 1947;45;57-73.

14. Cheung $C$, Ikram M, Sabanayagam C, Wong T, Retinal microvasculature as a model to study the manifestations of hypertension, Hypertension, 2012;60:1094-103.

15. Hammond S, Wells J, Marcus D, Prisant L, Ophthalmoscopic findings in malignant hypertension, $J$ Clin Hypertens (Greenwich), 2006;8:221-3. 